\title{
En quête d'index biologiques des maladies mentales
}

Tout être humain est
en perpétuelle
interaction avec son
environnement. Les
comportements qui
découlent de ces
interactions sont des
tentatives
d'adaptation aussi
harmonieuses que
possible à des situations
sans cesse renouvelées
et qui constituent les
expériences
existentielles. La
psychiatrie s'intéresse
au comportement
pathologique,
c'est-à-dire aux
tentatives
infructueuses
d'adaptation du sujet
à son milieu.

\section{ADRESSE}

E. Zarifian: Centre Esquirol, CHU Côte de

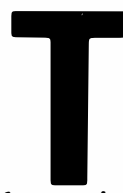

rois champs de références permettent d'aborder la psychopathologie : l'analyse du rôle de l'environnement qui est du ressort de la sociologie; l'étude de l'appareil psychique et des émotions qui appartient à la psychologie et aux sciences humaines; la psychiatrie biologique qui s'intéresse spécifiquement aux modifications du système nerveux central comme cause ou conséquence des troubles du comportement.

Toute maladie psychiatrique comporte vraisemblablement une origine plurifactorielle dont on retrouve, avec une pondération variable selon les cas, une origine dans les trois registres précédemment évoqués. Malheureusement, le cerveau est inaccessible à une observation directe chez l'homme vivant. Les efforts de la psychiatrie biologique au cours des vingt dernières années se sont donc portés vers l'identification d'index facilement accessibles à la périphérie et permettant de rendre compte de modifications biologiques dans le système nerveux central.

C'est cette quête d'index biologiques périphériques dans les maladies mentales nui a constitué jusqu'à aujourd'hui le domaine de la psychiatrie biologique.

\section{Champ actuel de la psychiatrie biologique}

Le concept d'index périphérique d'une maladie mentale repose sur une hypothèse simplificatrice. Toute anomalie du comportement implique une anomalie du fonctionnement cérébral. Cette anomalie, bio- logique par exemple, est inaccessible directement. Il sera donc nécessaire de mettre en évidence dans les liquides biologiques périphériques un témoin de l'anomalie centrale. Il serait souhaitable que cet index soit spécifique, retrouvé uniquement dans la pathologie étudiée et éventuellement symptomatique d'un désordre étiologique. Ce raisonnement est sujet à maintes critiques. En effet, si on retrouve dans le sang, le liquide céphalo-rachidien ou les urines, des catabolites des neurotransmetteurs centraux, il est difficile de les considérer comme un reflet fidèle et direct d'une éventuelle anomalie cérébrale. La barrière hémato-encéphalique contrôle les transferts entre le compartiment central et la périphérie. Les phénomènes dont la barrière hémato-encéphalique est le siège peuvent donc modifier quantitativement, indépendamment de l'anomalie cérébrale, les catabolites des neurotransmetteurs. En outre, ces neurotransmetteurs existent également à la périphérie et il sera très difficile de différéncier la part des catabolites venant du cerveau de celle venant de la périphérie.

C'est ainsi que l'on considère, d'une manière très approximative, que le catabolite principal de la noradrenaline, le MHPG (méthoxy-hydroxyphényl-glycol), que l'on peut doser à la périphérie, est un mélange issu du cerveau et de la périphérie dans une proportion d'environ 50 à $60 \%$ au bénéfice du cerveau.

Même si l'origine cérébrale du catabolite est prouvée, ce qui est loin d'être toujours le cas, le dosage ne renseigne ni sur la localisation anatomique de la modification biochi- 
mique, ni sur la nature exacte de cette modification. En effet, pour être schématique, on peut imaginer que la diminution du catabolite peut être due à un défaut de production ou à un excès de dégradation.

Une autre critique concerne l'utilisation du terme même de "marqueur ". Marqueur de quoi? Connaissant la complexité de fonctionnement du cerveau et les multiples interactions entre neurotransmetteurs, comment peut-on imaginer que l'anomalie d'un seul neurotransmetteur puisse être un index, c'est-à-dire, un témoin exclusif d'un comportement pyschopathologique complexe, comme un syndrome dépressif, ou une schizophrénie délirante. Il est évident que l'anomalie d'un seul neurotransmetteur ne peut pas être responsable de la richesse séméiologique des tableaux psychiatriques.

En dépit de ces réserves, de très nombreux travaux ont été publiés au cours de ces dernièrs années, tentant d'établir une corrélation entre une anomalie biologique et un trouble des comportements. Les axes de recherche ont finalement été guidés par des impératifs techniques. On s'est intéressé à ce qui était accessible, c'est-à-dire à ce qui était dosable.

Sans entrer dans le détail, on peut citer les principaux types d'investigations dans ce domaine: les monoamines cérébrales continuent à susciter des recherches importantes malgré la quasi absence de résultats positifs obtenus; les catabolites de la dopamine, de la noradrénaline, de la sérotonine, sont accessibles dans les milieux périphériques; les précurseurs de ces monoamines ont également été pris en considération ainsi que l'évaluation des enzymes de synthèse et de dégradation. Quelques travaux trop rares, et non comparables entre eux faute d'une méthodologie standardisée, concernent des études sur des pièces anatomiques post-mortem. Ces études biochimiques ne permettent pas, à l'heure actuelle, d'avoir une idée précise des mécanismes en cause dans les maladies mentales. La plaquette sanguine, facilement accessible, a beaucoup intéressé les psychiatres. Elle a même été considérée comme le modèle périphéri- que du neurone sérotoninergique. La plaquette est capable de capter la sérotonine, de la dégrader; en outre, les récepteurs plaquettaires sérotoninergiques et noradrénergiques peuvent être étudiés au moyen de ligands appropriés.

Parallèlement aux très nombreux travaux consacrés à l'étude des monoamines et des catabolites, différents systèmes hormonaux ont également été approchés : études statiques de concentrations hormonales ou études dynamiques d'axes hypothalamo-hypophysaires. Le test de freination à la déxaméthasone, le test de stimulation de l'hormone de croissance, le test de stimulation à la TSH (Thyroid Stimulating Hormone) font partie de la panoplie usuelle des psychiatres. Malheureusement, les résultats sont contradictoires et ne permettent pas de dégager un tableau biologique cohérent. Le pré-supposé théorique de toutes ces études ne peut plus être accepté par la neurobiologie contemporaine. Le cerveau neuronal n'implique pas une simple communication par câblage. Les interconnexions neuronales multiples, le nombre sans cesse croissant des neurotransmetteurs découverts, les fonctions complexes de la neuromodulation, doivent faire rejeter le schéma linéaire d'une communication neuronale par continuité, responsable à elle seule d'un comportement élaboré.

Le modèle du cerveau hormonal, qui implique une communication par réseaux, c'est-à-dire à distance, est plus proche des données actuelles de la neurobiologie. La libération de neurotransmetteurs au niveau des dendrites, et pas seulement au niveau des synapses, la fonction véritablement hormonale de certains neurotransmetteurs, impliquent une information excentrique globale de tout le cerveau.

En outre, cerveau et périphérie, même s'ils sont séparés par la barrière hémato-encéphalique, communiquent sans cesse selon un circuit à double canal. On sait par exemple que la même substance agit simultanément au niveau du cerveau et au niveau de la périphérie. L'angiotensine, au niveau cérébral, entraîne un comportement de soif; mais, à la périphérie, elle modifie le volume sanguin. La lulibérine, par son action cérébrale, module le comportement sexuel; mais, par son action périphérique, elle engendre l'ovulation. L'ocytocine induit un comportement maternel mais déclenche également la lactation. Cette complexité, nouvellement découverte, ne permet plus d'accepter le schéma simplificateur précédemment évoqué.

Quelques exemples qui avaient suscité de grands espoirs mais qui sont contestés actuellement, permettront d'illustrer les difficultés rencontrées par le concept d'index biologique périphérique en psychiatrie.

L'administration de dexaméthasone chez le sujet sain entraîne un effondrement de la cortisolémie. La découverte d'une absence de freination au test à la dexaméthasone avec maintien de cortisolémie normale, voire plus élevée que la normale, chez les mélancoliques endogènes, a fait croire pendant un temps qu'un marqueur biologique périphérique de la dépression avait été trouvé. En fait, cette anomalie de réponse au test de suppression à la déxaméthasone n'a rien de spécifique. On le retrouve perturbé lors de pertes de poids importantes, chez les éthyliques, chez les patients prenant des benzodiazépines. C'est sans doute le marqueur d'un changement physiologique associé au syndrome dépressif, mais ce n'est pas un marqueur étiologique de la dépression mélancolique elle-même.

En 1984, Winokur signalait une augmentation significative des polynucléaires neutrophiles et une diminution des lymphocytes dans les dépressions mélancoliques. En fait, il s'agissait de la conséquence d'une augmentation de la cortisolémie qui est retrouvée de manière quasiconstante dans la dépression. Or, tout stress augmente le cortisol. Là encore, il s'agit d'une anomalie non spécifique, l'augmentation du cortisol engendrant une deuxième modification non spécifique de la dépression, l'altération de la formule sanguine.

On pourrait reprendre point par point les différents index biologiques périphériques qui ont été avancés comme des marqueurs de maladies mentales et faire une critique systématique des résultats obtenus. Les données de la littérature 
sont souvent contradictoires, voir: difficilement reproductibles. Des raisons méthodologiques se surajoutent aux arguments précédemment énoncés pour expliquer les discordances de résultats. Les techniques de mesures ne sont pas standardisées, les conditions de recueil et de transport des échantillons ne sont pas précisées, les facteurs de l'environnement pouvant influencer les résultats (comme l'heure du prélèvement, le moment de l'année) et les caractéristiques individuelles (comme l'âge, le sexe, le poids) ne sont pas toujours pris en compte. En outre, les groupes contrôles sont très difficiles à réaliser en psychiatrie. Si l'on s'adresse à des volontaires, un biais peut exister car ils ne partagent pas les conditions de vie des hospitalisés. Si l'on s'adresse à une autre pathologie, on introduit sûrement un biais non maîtrisable. En outre, une modification biologique doit-elle être corrélée à un syndrome, c'est-à-dire à une anomalie complexe du comportement, ou à un symptôme constitutif du syndrome mais qui, dans ce cas, a rarement une spécificité.

Le ralentissement moteur est certes fréquent mais non constant dans la dépression, et il existe également dans d'autres pathologies, psychiatriques ou non.

\section{Vers un renouveau en psychiatrie biologique}

Une autre difficulté, et non des moindres dans toutes tentatives de corréler une anomalie biologique à un trouble du comportement, concerne la réalisation de groupes homogènes de malades. Les critères diagnostiques en psychiatrie sont encore sujets à caution. Il n'existe pas de consensus au plan international pour définir clairement les malades. Il est donc illusoire de vouloir comparer les travaux issus de différents pays, voire de différentes écoles. Dans certains cas, l'étude longitudinale de malades isolés pourrait peut-être être plus fructueuse que l'étude de groupes hétérogènes. Certaines maladies cycliques comme la psychose maniaco-dépressive qui réalisent au fil du temps, chez le même sujet, trois états possibles : la normalité, la mé- lancolie et l'état maniaque, permettent de prendre le sujet comme son propre témoin. En outre, une standardisation des méthodologies et des techniques de dosages faciliterait l'interprétation des résultats.

Une voie nouvelle semble également s'ouvrir avec le couplage de l'évaluation simultanée de différents marqueurs. En effet, malgré les travaux très nombreux concernant les évaluations hormonales au cours des troubles de l'humeur, il faut remarquer que très peu de travaux se sont intéressés simultanément à l'évaluation d'un profil endocrinien. Les quelques résultats récemment publiés, prenant en compte cette lacune, semblent plus prometteurs que les travaux qui s'intéressaient uniquement à un type d'hormone donné.

Si la nosologie psychiatrique classique est impuissante à constituer des groupes homogènes et à distinguer des patients entre eux, il existe peut-être des moyens de contourner cette difficulté. Certains groupes commencent à quantifier les processus cognitifs et les fonctions supérieures, indépendamment de toute approche nosologique. L'étude comparative des fonctions cognitives entre l'état normal et l'état pathologique semble apporter plus d'informations que les données de la clinique classique.

Une autre approche consiste à déterminer des groupes homogènes de patients sur la base de marqueurs biologiques déjà connus et n'ayant rien à voir avec les neurotransmetteurs. C'est ainsi qu'au sein d'une population de malades considérée comme homogène selon les critères cliniques classiques, la typologie des groupes sanguins, des groupes HLA, l'étude des hémoglobines et de l'haptoglobine, permet une distinction que la clinique ne permettait pas.

Enfin, de nouveaux outils viennent aider au développement de la psychiatrie biologique. L'imagerie cérébrale permet un abord direct du cerveau chez l'homme vivant et d'une manière totalement atraumatique. La résonance magnétique nucléaire $(\mathrm{RMN})$ en est encore à un stade trop précoce de développement pour que des résultats puissent même être évoqués.
Par contre, l'utilisation de la caméra à positons a déjà apporté une série d'informations concernant le métabolisme cérébral et ses variations au cours de certaines maladies mentales. Cette technique permet également une visualisation des récepteurs cérébraux et une étude de l'interaction entre les psychotropes usuels et ces récepteurs.

Il est également possible que la génétique moléculaire puisse venir en aide à la psychiatrie biologique. L'isolement récent du gène codant pour la tyrosine hydroxylase et l'étude de son polymorphisme au sein des populations est une approche originale du métabolisme des catécholamines.

En conclusion, si l'approche traditionnelle de recherche des index biologiques périphériques des maladies mentales s'est avérée globalement peu fructueuse compte tenu de l'importance des efforts mis en œuvre, de nouvelles approches du fonctionnement cérébral chez l'homme vivant paraissent plus rationnelles et plus prometteuses

\section{REFERENCES}

Langer SZ, Raisman R, Sechter D, Gay C, Loo $\mathrm{H}$, Zarifian E. ${ }^{3} \mathrm{H}$-imipramine and ${ }^{3} \mathrm{H}$ desipramine binding sites in depression. In : Usdin E, Asbcrg M, Bcrtilsson L, Sjoqvist F, cds. Frontiers in Biochemical and Pharmacological Research in Depression (adv in Biochem Psychopharmacol, vol.39). New York: Raven Press, 1984: $113-25$.

Baron JC, Comar D, Zarifian E, et al. Dopaminergic receptor sites in human brain: positron emission tomography. Neurology 1985; 35: 16-24.

Usdin E, Mandell A J. Biochemistry of mental disorders. New vistas. New York: Marcel Dekker, $1978: 268$.

Mendels J, Amsterdam JD. The psychobiology of affective disorders. Bascl: Karger, 1980: 219.

Baxter CF, Melnechuk T. Perspectives in schizophrenia research. New York: Raven Press, 1980: 444 .

\section{TIRES A PART}

E. Zarifian : Centre Esquirol, CHU Côte de Nacre, 14033 Caen Cedex. 